\title{
Anatomy of Language Impairments in Primary Progressive Aphasia
}

\author{
Emily Rogalski, ${ }^{1}$ Derin Cobia, ${ }^{2}$ Theresa M. Harrison, ${ }^{1}$ Christina Wieneke, ${ }^{1}$ Cynthia K Thompson, ${ }^{1,3,4}$ \\ Sandra Weintraub, ${ }^{1,2}$ and M.-Marsel Mesulam ${ }^{1,4}$ \\ ${ }^{1}$ Cognitive Neurology and Alzheimer's Disease Center, Northwestern University (NU) Feinberg School of Medicine, Chicago, Illinois 60611, ${ }^{2}$ NU Feinberg \\ School of Medicine, Department of Psychiatry and Behavioral Sciences, Chicago, Illinois 60611, ${ }^{3}$ Department of Communication Sciences and Disorders, \\ Northwestern University, Evanston, Illinois 60208, and ${ }^{4}$ NU Feinberg School of Medicine, Department of Neurology, Chicago, Illinois 60611
}

Primary progressive aphasia (PPA) is a clinical dementia syndrome characterized by progressive decline in language function but relative sparing of other cognitive domains. There are three recognized PPA variants: agrammatic, semantic, and logopenic. Although each PPA subtype is characterized by the nature of the principal deficit, individual patients frequently display subtle impairments in additional language domains. The present study investigated the distribution of atrophy related to performance in specific language domains (i.e., grammatical processing, semantic processing, fluency, and sentence repetition) across PPA variants to better understand the anatomical substrates of language. Results showed regionally specific relationships, primarily in the left hemisphere, between atrophy and impairments in language performance. Most notable was the neuroanatomical distinction between fluency and grammatical processing. Poor fluency was associated with regions dorsal to the traditional boundaries of Broca's area in the inferior frontal sulcus and the posterior middle frontal gyrus, whereas grammatical processing was associated with more widespread atrophy, including the inferior frontal gyrus and supramarginal gyrus. Repetition performance was correlated with atrophy in the posterior superior temporal gyrus. The correlation of atrophy with semantic processing impairment was localized to the anterior temporal poles. Atrophy patterns were more closely correlated with domain-specific performance than with subtype. These results show that PPA reflects a selective disruption of the language network as a whole, with no rigid boundaries between subtypes. Further, these atrophy patterns reveal anatomical correlates of language that could not have been surmised in patients with aphasia resulting from cerebrovascular lesions.

\section{Introduction}

Primary progressive aphasia (PPA) is increasingly recognized as a language-based clinical dementia syndrome (Mesulam, 2003). At its initial stages, the syndrome is remarkable in its predilection for the left hemisphere language network, a feature that has been well documented with clinical and quantitative imaging methods (e.g., cortical thickness mapping) (Mesulam et al., 2009a; Rohrer et al., $2009,2010)$. The partial and progressive breakdown of language functioning as a result of neurodegeneration in PPA offers opportunities for probing the neuroanatomical substrates of language with an approach that complements and extends the clinicopathological correlations found in patients with stroke-related aphasia.

Classic aphasiology, based primarily on cerebrovascular lesions, has shown that aphasias can differ in their clinical manifes-

Received 0ct. 22, 2010; revised Dec. 1, 2010; accepted Jan. 2, 2011.

This work was supported by Grants DC008552 from the National Institute on Deafness and Communication Disorders, AG13854 (to the Alzheimer Disease Center) from the National Institute on Aging, and 5KL2RR025740 from the National Center for Research Resources. The content of this manuscript is solely the responsibility of the authors and does not necessarily represent the official views of the National Center for Research Resources or the National Institutes of Health. Imaging was performed at the Northwestern University Department of Radiology Center for Advanced MRI.

The authors declare no competing financial interests.

Correspondence should be addressed to Dr. Emily J. Rogalski, Northwestern University, Cognitive Neurology and Alzheimer's Disease Center, 320 E Superior Street, Searle Building 11th Floor, Chicago, IL 60611. E-mail: erogalski@gmail.com.

DOI:10.1523/JNEUROSCI.5544-10.2011

Copyright $\odot 2011$ the authors $\quad 0270-6474 / 11 / 313344-07 \$ 15.00 / 0$ tations depending on lesion location within the language network. Similar clinicopathological correlations can be identified in PPA. However, these correlations in PPA are more complex than those in patients with stroke because even sites of pronounced atrophy in PPA contain residual neurons that continue to participate in language functions (Sonty et al., 2003). Based on the nature of the language impairment, three PPA variants have been identified [agrammatic (PPA-G), semantic (PPA-S), and logopenic (PPA-L)], each associated with a clinically concordant distribution of peak cortical atrophy (Gorno-Tempini et al., 2004; Mesulam et al., 2009a; Rohrer et al., 2009).

Although each subtype is characterized by the nature of the principal deficit, individual PPA patients frequently display less severe abnormalities in additional language functions. An alternative approach for investigating the neural correlates of each PPA subtype is to examine relationships between the distribution of atrophy and specific language dimensions (e.g., grammatical processing or fluency) across PPA variants. Using this strategy, atrophy and performance operate as continuous variables without the creation of discrete groupings.

Advances in computational anatomy have enabled such analyses by quantitatively computing relationships between MRIderived estimates of atrophy and cognitive performance. Specifically, semiautomated surface-based methods have been developed to allow for detection of changes in cortical thickness on a vertex-by-vertex basis with subvoxel resolution (Fischl et al., 


\section{Table 1. Neuropsychological features of PPA variants}

PPA-G The central feature is an abnormality of syntax (word-order) or some other aspect of
grammar in spoken or written language in the presence of relatively
preserved single word comprehension. Fluency is often impaired.
PPA-S The central feature is an abnormality of single word comprehension in the presence
of relatively preserved grammar and fluency. Output is circumlocutory, occasionally
uninformative and frequently paraphasic. Naming is severely impaired.
PPA-L The central feature is intermittent word-finding hesitations and phonemic
paraphasias. Naming is impaired but not as severely as in PPA-S and improves
upon phonemic cueing.

1999; Fischl and Dale, 2000). These methods have been successful for identifying relationships between regional cortical thickness measures and intelligence quotient (Shaw et al., 2006), personality measures (Wright et al., 2006, 2007), and memory in healthy individuals (Walhovd et al., 2006).

The present study used this computational anatomy approach to investigate the patterns of cortical thinning (i.e., atrophy) associated with cognitive performance across four domains of language, namely auditory comprehension of single words (semantic processing), sentence repetition, production of complex sentences (grammatical processing), and speech fluency. The two goals were to probe the anatomical substrate of language with a novel approach and to explore the relationship between discrete clinical subtypes of PPA and dimensions of language.

\section{Materials and Methods}

\section{Participants}

Thirty-one consecutive patients with a root diagnosis of PPA (ages, $48-84$ years) and 27 cognitively healthy controls of similar age and education level participated in the study. The diagnosis of PPA was made on the basis of an isolated and progressive language impairment, according to established guidelines. Participants were recruited from the Primary Progressive Aphasia Program, funded by the National Institute on Deafness and Other Communication Disorders, and evaluated at the Cognitive Neurology and Alzheimer's Disease Center and the Aphasia and Neurolinguistics Research Laboratory, both at Northwestern University and the Feinberg School of Medicine.

All of the patients received a descriptive clinical diagnosis of PPA-G, PPA-L, or PPA-S based on evaluations by clinicians with extensive experience with this disease (M.M.M. and S.W.) and following the established guidelines (Table 1). Two patients resisted easy classification into one of the three subtypes. One presented with grammatical impairments as well as single-word auditory comprehension deficits and was given a mixed subtype designation, as suggested in a previous publication (Mesulam et al., 2009a). The second patient demonstrated features consistent with PPA-L but also evinced mild syntactic deficits, and was given a PPA-G/L designation.

Table 2 presents demographic information and clinical aphasia scores for each patient, as well as mean scores for the patient and control groups. The mean age of patients with PPA was $63.0( \pm 8.2)$ years and mean education level was $16.2( \pm 2.5)$ years; $38.7 \%$ of the sample were male. Patients and controls did not statistically differ by age, education, or gender distribution. The mean duration of reported symptoms in the PPA group was $3.4( \pm 1.5)$ years. All participants were right-handed. Informed consent was obtained from all participants and the Institutional Review Board of Northwestern University approved the study.

\section{Neuropsychological measures}

Semantic processing. We used a test of auditory single-word processing as a measure of lexical-semantic processing: a 36 -item subset of moderately difficult items (items 157-192) from the fourth edition of the Peabody Picture Vocabulary Test (PPVT-IV) (Dunn and Dunn, 2006). For this test, the patient is required to match an auditory word representing a living or nonliving object, action, adjective, scene, or attribute to one of four picture choices. This test measures general semantic processing abilities across categories. We did not compare category-specific deficits (e.g., living things vs artifacts). Performance on this subset of items from the PPVT-IV was used in a previous study to successfully differentiate PPA patients with and without comprehension deficits (Mesulam et al., 2009a). This subset of items was recommended because the difficulty level of the items is likely to avoid floor or ceiling effects. Scores are reported as percentage correct.

Sentence repetition. The sentence items on the Repetition subtest of the Western Aphasia Battery (WAB; items 10-15) (Kertesz, 1982), which requires individuals to repeat sentences, was used. Single word and sentence repetition performance can show double dissociations, suggesting there may be different mechanisms underlying these processes (McCarthy and Warrington, 1987). To avoid this confound, we restricted our analysis to the sentence items of the WAB Repetition subtest. Scores are reported as percentage correct.

Grammatical processing. We used a measure of syntactically complex and simple sentence production, the Northwestern Anagram Test (NAT; http://www.soc.northwestern.edu/NorthwesternAnagramTest/) as a measure of grammatical processing. A subset of 10 items from the NAT that measure object-extracted who-questions and subject-extracted who-questions was chosen for this analysis, since it is highly correlated with other measures of sentence production (Weintraub et al., 2009). In this test, the patient is asked to order single words, each printed on a separate card, to correctly depict the action in a target picture. The NAT was developed to test the ability to order words into a grammatically accurate sentence. It was specifically designed for patients who present with speech production, word comprehension, and/or word-finding difficulties, or reduced working memory capacity, who may not be able to demonstrate grammatical competence with measures of spontaneous production or sentence comprehension. Scores are reported as percentage correct.

Fluency. We recorded speech samples from each of our patients while they told the story of Cinderella from a wordless picture book. The speech samples were transcribed and segmented into utterances based on syntactic, prosodic, and semantic criteria by neurolinguists in the Aphasia and Neurolinguistics Research Laboratory. Each utterance was coded and analyzed using a previously published procedure that quantifies both lexical and structural detail (Thompson et al., 1995, 1997). Mean length of utterance in words was used as the metric of fluency for this study, since it accounts for not only speed of verbal output, but word-finding difficulty and pauses.

\section{MRI acquisition parameters}

T1-weighted three-dimensional MP-RAGE sequences (repetition time, $2300 \mathrm{~ms}$; echo time, $2.86 \mathrm{~ms}$; flip angle, $9^{\circ}$; field of view, $256 \mathrm{~mm}$ ) recording 160 slices at a slice thickness of $1.0 \mathrm{~mm}$ were acquired on a 3T Siemens TRIO system using a 12-channel birdcage head coil. Imaging was performed at the Northwestern University Department of Radiology Center for Advanced MRI.

\section{Image processing and cortical thickness measurement}

MR images were processed using the image analysis suite FreeSurfer (version 4.5.0), which is documented and freely available for download online (http://surfer.nmr.mgh.harvard.edu/). Cortical thickness estimates were calculated by measuring the distance between representations of the white-gray and pial-CSF boundaries across each point of the cortical surface. The technical details of these procedures were described previously (Fischl and Dale, 2000). This method was demonstrated to be reliable across scanning protocols and platforms in a series of healthy elderly participants when examining general cognitive performance and cortical thickness (Dickerson et al., 2008).

\section{Statistical analysis}

First, statistical surface maps were generated using a general linear model (GLM) that displayed differences in cortical thickness between the PPA and healthy groups for each vertex along surface representations of the entire neocortex.

Second, to assess the relationship between cortical thinning (atrophy) and cognitive performance within the PPA group, four separate statisti- 
Table 2. Demographic and neuropsychological scores for PPA patients and controls

\begin{tabular}{|c|c|c|c|c|c|c|c|c|c|}
\hline Participant & Gender & PPA subtype & Age & WAB AQ (\%) & BNT (\%) & PPVT (\%) & WAB sentence repetition (\%) & NAT - $10(\%)$ & MLU words \\
\hline $\mathrm{CH} 10$ & $\mathrm{~F}$ & G & 57 & 76.7 & 68.3 & 94.4 & 54.5 & 50 & - \\
\hline P13 & M & G & 62 & 82.3 & 98.3 & 100 & 72.7 & 50 & 4.0 \\
\hline P14 & M & G & 59 & 79.9 & 81.7 & 94.4 & 66.7 & 40 & 5.0 \\
\hline P20 & $\mathrm{F}$ & G & 84 & 61.1 & 70.0 & 83.3 & 47.0 & - & - \\
\hline P23 & $\mathrm{F}$ & G & 56 & 41.8 & 26.7 & 83.3 & 25.8 & 10 & - \\
\hline P27 & $\mathrm{F}$ & G & 61 & 75.3 & 88.3 & 100 & 81.8 & 50 & 5.4 \\
\hline P31 & $M$ & G & 72 & 80.6 & 73.3 & 88.9 & 40.9 & 50 & 11.3 \\
\hline P32 & $\mathrm{F}$ & G & 68 & 63.5 & 28.3 & 86.1 & 27.3 & - & 5.9 \\
\hline P34 & $\mathrm{F}$ & G & 62 & 73.9 & 45.0 & 94.4 & 34.8 & 50 & 7.1 \\
\hline P36 & $\mathrm{F}$ & G & 69 & 85.0 & 93.3 & 88.9 & 83.3 & 50 & 6.8 \\
\hline P39 & M & G & 70 & 78.1 & 71.7 & 91.7 & 66.7 & 40 & - \\
\hline P41 & $\mathrm{F}$ & $\mathrm{G} / \mathrm{L}$ & 57 & 88.0 & 81.7 & 91.7 & 75.8 & 60 & - \\
\hline P1 & $M$ & $\mathrm{~L}$ & 69 & 92.0 & 98.3 & 97.2 & 84.8 & 90 & 9.5 \\
\hline P16 & $M$ & $\mathrm{~L}$ & 58 & 86.9 & 90.0 & 97.2 & 84.8 & 90 & 10.5 \\
\hline P17 & $\mathrm{F}$ & $\mathrm{L}$ & 65 & 78.6 & 83.3 & 83.3 & 81.8 & 70 & 5.4 \\
\hline P22 & $\mathrm{F}$ & $\mathrm{L}$ & 75 & 97.2 & 88.3 & 97.2 & 87.9 & 100 & 13.9 \\
\hline P25 & $M$ & $L$ & 60 & 93.2 & 98.3 & 100 & 83.3 & 70 & 8.6 \\
\hline P26 & $M$ & $\mathrm{~L}$ & 48 & 83.2 & 98.3 & 100 & 84.8 & 100 & 9.2 \\
\hline P29 & $M$ & $\mathrm{~L}$ & 62 & 95.8 & 40.0 & 97.2 & 100 & 100 & - \\
\hline P30 & $F$ & $L$ & 75 & 88.8 & 83.3 & 97.2 & 60.6 & 100 & 10.7 \\
\hline P35 & $\mathrm{F}$ & $\mathrm{L}$ & 76 & 87.6 & 93.3 & 72.2 & 68.2 & 90 & 6.1 \\
\hline P37 & $M$ & $\mathrm{~L}$ & 61 & 95.2 & 96.7 & 100 & 97.0 & 60 & - \\
\hline P40 & $F$ & $L$ & 57 & 95.4 & 90.0 & 100.0 & 81.8 & 100 & - \\
\hline CH5 & $\mathrm{F}$ & $S$ & 64 & 80.6 & 8.3 & 38.9 & 81.8 & 100 & - \\
\hline $\mathrm{CH} 6$ & $\mathrm{~F}$ & $S$ & 56 & 75.5 & 5.0 & 38.9 & 75.6 & 100 & - \\
\hline $\mathrm{CH} 7$ & $F$ & $S$ & 53 & 85.6 & 10.0 & - & 97.0 & 100 & - \\
\hline $\mathrm{CH} 9$ & $\mathrm{~F}$ & $S$ & 55 & 95.0 & 61.7 & 50 & 97.0 & 100 & - \\
\hline P21 & $M$ & $S$ & 63 & 88.2 & 23.3 & 47.2 & 95.5 & 100 & 13.7 \\
\hline P24 & $\mathrm{F}$ & $S$ & 53 & 65.9 & 6.7 & 22.2 & 60.6 & 60 & 7.8 \\
\hline P28 & $\mathrm{F}$ & $S$ & 54 & 83.2 & 10.0 & 27.8 & 86.4 & 100 & 10.6 \\
\hline P38 & $\mathrm{F}$ & Mixed & 70 & 71.8 & 15.0 & 47.2 & 62.1 & 20 & - \\
\hline \multirow[t]{2}{*}{ PPA } & Mean & & 62.9 & 81.5 & 62.1 & 80.4 & 72.5 & 72.4 & 8.4 \\
\hline & Standard deviation & & 8.2 & 12.0 & 34.1 & 24.6 & 20.5 & 27.7 & 3.0 \\
\hline \multirow[t]{2}{*}{ Normal controls } & Mean & & 62.3 & 99.7 & 96.9 & 97.9 & 98.3 & 98.3 & 11.2 \\
\hline & Standard deviation & & 6.6 & 0.7 & 3.1 & 3.8 & 3.2 & 4.8 & 2.1 \\
\hline
\end{tabular}

WAB AQ, Western Aphasia Battery Aphasia Quotient; BNT, Boston Naming Test; WAB sentence repetition, Items 10 - 15 of the repetition subtest of the WAB; NAT-10, subset of 10 items from the NAT; MLU, mean length of utterance;-no completed; (\%), percentage correct; $F$, female; $M$, male.

cal GLM surface maps were computed to identify significant relationships between cortical thickness of the entire cortical hemisphere and each language measure described above. The false discovery rate (FDR), which controls for the expected proportion of false positives in a statistical test, was applied at 0.05 (unless otherwise stated) to adjust for multiple comparisons (Genovese et al., 2002).

Once cortical areas with significant effects were identified from the entire hemisphere, a region of interest (ROI)-based approach was used to further investigate quantitative relationships (Dickerson et al., 2008). ROIs included all relevant vertices in which significant thickness-performance correlations were observed. Associations spanned across more than one anatomical region for some comparisons in the whole-brain analysis (e.g., inferior frontal gyrus and sensory motor cortex). In this case, regions were divided into two or more ROIs based on the anatomical landmarks provided in FreeSurfer. ROIs were first delineated on a template cortical surface and then registered from the template surface space to each individual subject's surface. At this point, mean cortical thickness was calculated for each ROI. Pearson bivariate correlation analyses were used to detect the strength of the correlation between the mean thickness of the ROI and the language measure of interest. Statistical analyses were performed using PASW 18.0 (SPSS).

\section{Results}

Cortical thinning in PPA patients compared with controls

Results from the GLM analysis in Figure 1 show the regional distribution of cortical thinning (atrophy) of the PPA patients compared with controls (FDR, 0.01). Consistent with previous work, cortical thinning was asymmetric and most severe in the perisylvian language regions of the left cerebral hemisphere. This included major involvement of the entire left temporal lobe, as well as the supramarginal and angular gyri. Significant atrophy was also noted across the inferior frontal lobe and insular regions. Although not as prominent as in the left hemisphere, thinning in the right hemisphere was primarily noted in the supramarginal gyrus and banks of the superior temporal sulcus.

\section{Cortical thickness correlates of semantic processing (auditory single-word comprehension)}

Utilizing GLM statistical surface maps corrected for multiple comparisons (FDR, 0.05), PPVT-IV performance was significantly correlated with atrophy in the temporal pole (bilaterally), including anterior portions of the superior, middle, and inferior temporal gyri (Fig. 2). Secondary analysis of this ROI revealed that the strength of the correlation was greater in the left hemisphere $(r=0.89, p<0.001)$ than the right hemisphere $(r=0.79$, $p<0.001$ ) (Table 3), although both were significant.

\section{Cortical thickness correlates of sentence repetition}

Corrected statistical surface maps (FDR, 0.05) revealed a single region located in the medial aspect of the posterior superior temporal gyrus (transverse temporal or Heschl's gyrus) that demon- 

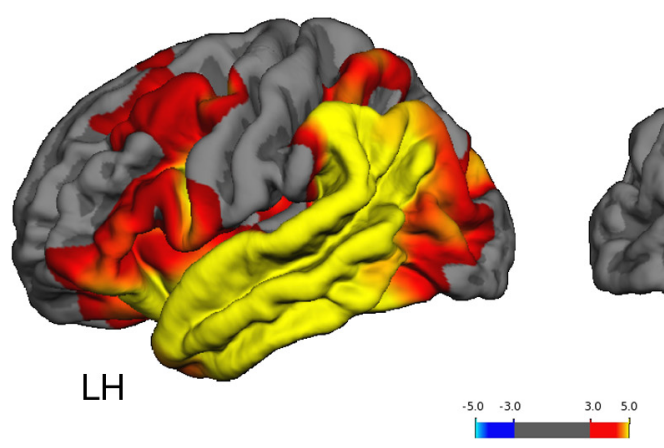

Figure 1. Distribution of cortical thinning on the pial surface in PPA patients compared with a control group. Significance is displayed as a $\log _{(10)} p$ value. LH, Left hemisphere; $\mathrm{RH}$, right hemisphere.
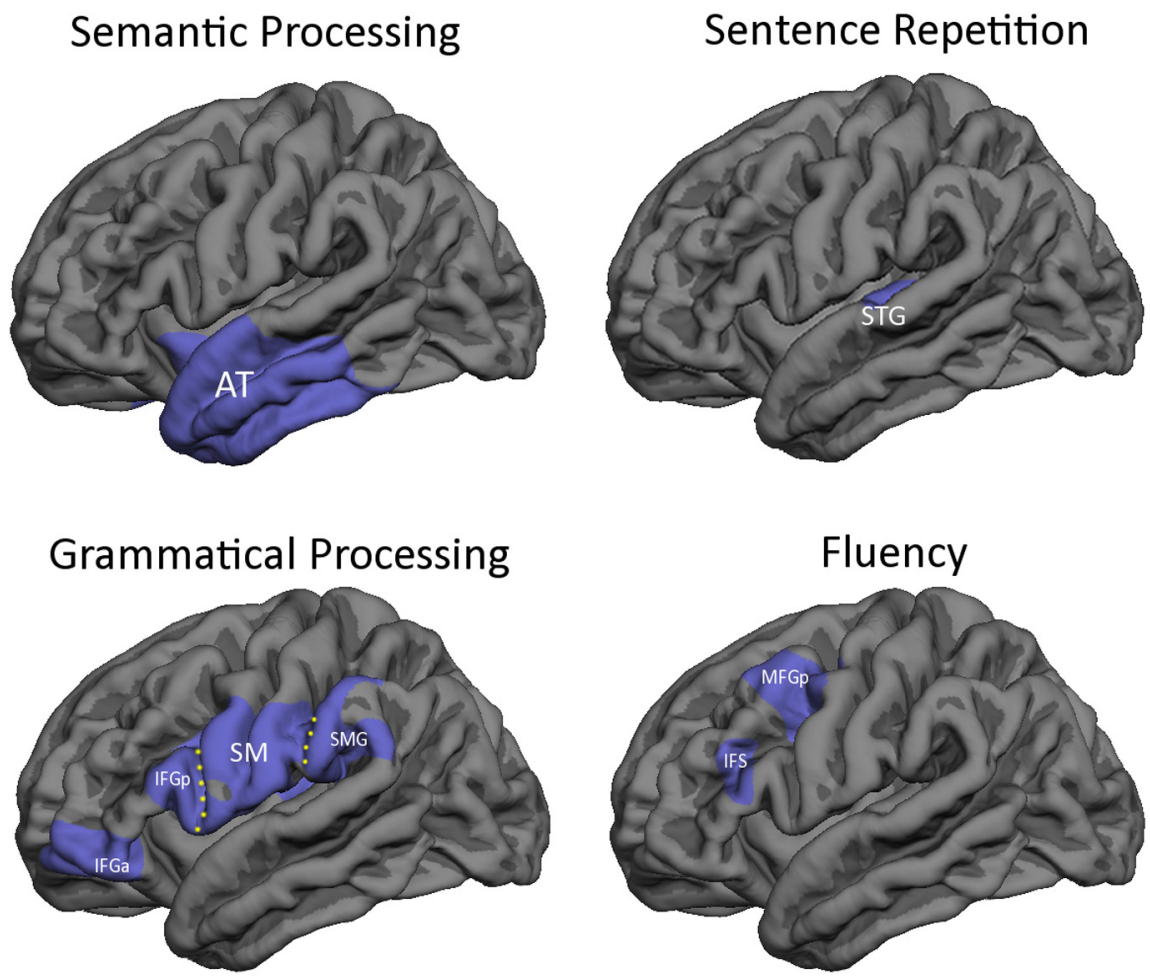

Figure 2. Colored areas highlight regions on the lateral left hemisphere pial surface that demonstrate a significant correlation between cortical thickness and semantic processing (top left), repetition (top right), grammatical processing (bottom left), and fluency measures (bottom right). AT, Anterior temporal; STG, superior temporal gyrus; IFGa, anterior IFG; IFGp, posterior IFG; SM, sensory motor cortex; SMG, supramarginal gyrus; IFS, inferior frontal sulcus; MFGp, posterior middle frontal gyrus. The yellow dotted lines indicate the approximate borders between regions of interest.

strated significant correlations in the left hemisphere between thickness and sentence repetition performance. This was quantitatively confirmed in the ROI analysis $(r=0.65, p<0.001)$ (Fig. 2, Table 3).

\section{Cortical thickness correlates of grammatical processing (sentence production)}

The significant NAT performance correlates were restricted to the left hemisphere. Compared with other language measures, the regional distribution of significant thickness-performance relationships was more widespread in the NAT, and included areas on both the medial and lateral aspects of the brain. Four regions were identified on the lateral surface of the corrected statistical surface maps (FDR, 0.05), one encompassing the anterior aspects of the inferior frontal gyrus (IFG), a second in the posterior IFG, a third in sensory motor cortex, and a fourth in the inferior parietal lobule, encompassing the supramarginal gyrus (Fig. 2, Table 3). Significant correlates on the medial surface included the superior frontal gyrus (SFG), paracentral lobule, and the precuneus (Table 3 ). The strength of these correlations were quantified and confirmed during ROI analyses.

\section{Cortical thickness correlates of word fluency}

Four regions (two in the left hemisphere and two in the right) on the statistical surface maps were significantly correlated with fluency based on the mean length of utterance ( $p=0.01$, FDR uncorrected): the posterior aspect of the middle frontal gyrus, bilaterally; the left hemisphere inferior frontal sulcus, and right hemisphere SFG (Fig. 2). The strength of these correlations ranged from $p=0.014$ to $p<$ 0.001 (Table 3).

\section{Analysis of performance and cortical thickness by subtype}

Individual patient scores for each language measure and thickness data were plotted and coded by subtype to determine whether these correlations aligned with subtyping distinctions (Fig. 3). The PPA-S patients could be clearly differentiated from the PPA-G and PPA-L patients based on single-word comprehension performance (using 60\% correct as a cutoff on the PPVT) and cortical thickness of the anterior temporal pole (using 2 as a the cutoff for mean thickness) (Fig. 3). Likewise, PPA-G patients could be separated from PPA-S and PPA-L patients based on NAT performance $(<60 \%$ correct) (Fig. 3). However, thickness measurements in the anterior inferior frontal gyrus could not differentiate the PPA-G from the other PPA variants (Fig. 3). Repetition and fluency performance abnormalities did not emerge as primary deficits for a

single PPA variant.

\section{Discussion}

The present study investigated patterns of cortical thinning along the entire cerebral cortex in an unselected group of consecutively enrolled PPA patients to determine the location of atrophy most related to performance in four distinct aspects of language: semantic processing (auditory single-word comprehension), repetition, grammatical processing (sentence production), and fluency. Compared with controls, the PPA group as a whole showed widespread atrophy affecting the entire left hemisphere language network and additional areas of the temporal, frontal and parietal lobes (Fig. 1). Results from our thickness and cognition correlation analyses demonstrate that each atrophic component is differentially related to performance on specific language 
tasks, such that performance on our measures of grammatical processing and repetition were related to left hemisphere perisylvian regions, whereas fluency and semantic processing demonstrated bilateral associations (Fig. 2).

One of the most interesting findings of this study is the neuroanatomical distinction between fluency (i.e., mean length of utterance) impairments from those of grammatical processing, as measured by sentence production ability. In classic aphasiology, the term "nonfluent" is used to refer to patients' reduced phrase length, diminished words per minute, or agrammatic sentence production (Hillis, 2007). However, in our work with PPA, we have cautioned against using the term "nonfluent" as a surrogate for "agrammatic" because fluency and grammatical ability can be dissociated. That is, reduced fluency can be seen in the agrammatic subtype, in the logopenic subtype, and in patients with a nonaphasic apraxia of speech; whereas impaired grammatical ability as reflected in difficulty producing sentences is a more specific finding that we have identified as the defining feature of the PPA-G subtype of PPA (Mesulam et al., 2009a). The present findings extend the behavioral dissociations between components of grammatical ability and fluency by demonstrating neuroanatomical distinctions.

The present results show that poor fluency was most closely associated with neuronal loss in areas dorsal to the traditional boundaries of Broca's area and also with similar regions in the right hemisphere. These areas, in the banks of the inferior frontal sulcus and the posterior part of the middle frontal gyrus, represent premotor cortices and may reflect the principally motor planning origins of poor fluency (Fig. 2). This is not to say that atrophy in other parts of the left hemisphere may not contribute to decreased fluency, but it does suggest that its principal correlate in PPA is located in premotor areas and that this is distinct from principal atrophy sites associated with the ability to construct grammatically correct sentences, which were located in a distributed set of left hemisphere regions, including portions of the inferior frontal gyrus and the supramarginal gyrus, two core components of the language network (Fig. 2, Table 3).

The ability to repeat sentences was associated with cortical thickness of the posterior aspect of the superior temporal gyrus. In patients with aphasia caused by stroke, the conduction aphasia subtype is characterized by a primary deficit in repetition classically thought to arise from damage to the left arcuate fasciculus, which results in a disconnection of the temporal and frontal lobes (Lichtheim, 1885; Damasio and Damasio, 1980). Recently, it has been suggested that damage to cortical gray matter in the left inferior parietal lobe is sufficient to produce repetition deficits (Zatorre et al., 1992; Anderson et al., 1999; Hickok et al., 2000; Fridriksson et al., 2010). In our group of PPA patients, sentence repetition scores utterance.
Table 3. Relationship between cortical thickness and neuropsychological performance

\begin{tabular}{|c|c|c|c|}
\hline $\mathrm{ROI}$ & Hemisphere & $r$ & $p$ value \\
\hline \multicolumn{4}{|l|}{ Semantic Processing } \\
\hline Temporal pole & $\mathrm{R}$ & 0.79 & $<0.001$ \\
\hline Temporal pole & L & 0.89 & $<0.001$ \\
\hline \multicolumn{4}{|l|}{ Sentence Repetition } \\
\hline Superior temporal gyrus, posterior & L & 0.65 & $<0.001$ \\
\hline \multicolumn{4}{|l|}{ Grammatical Processing } \\
\hline Inferior frontal gyrus, anterior & L & 0.68 & $<0.001$ \\
\hline Inferior frontal gyrus, posterior & $\mathrm{L}$ & 0.56 & 0.002 \\
\hline Sensory motor cortex & $\mathrm{L}$ & 0.75 & $<0.001$ \\
\hline Supramarginal gyrus & L & 0.62 & $<0.001$ \\
\hline \multicolumn{4}{|c|}{ Superior frontal gyrus, medial and paracentral } \\
\hline lobule & L & 0.70 & $<0.001$ \\
\hline Precuneus & L & 0.68 & $<0.001$ \\
\hline \multicolumn{4}{|l|}{ Fluency } \\
\hline Inferior frontal sulcus & L & 0.70 & 0.001 \\
\hline Middle frontal gyrus, posterior & $\mathrm{L}$ & 0.76 & $<0.001$ \\
\hline Middle frontal gyrus, posterior & $\mathrm{R}$ & 0.57 & 0.014 \\
\hline Superior frontal gyrus & $\mathrm{R}$ & 0.77 & $<0.001$ \\
\hline
\end{tabular}

R, Right; L, left.
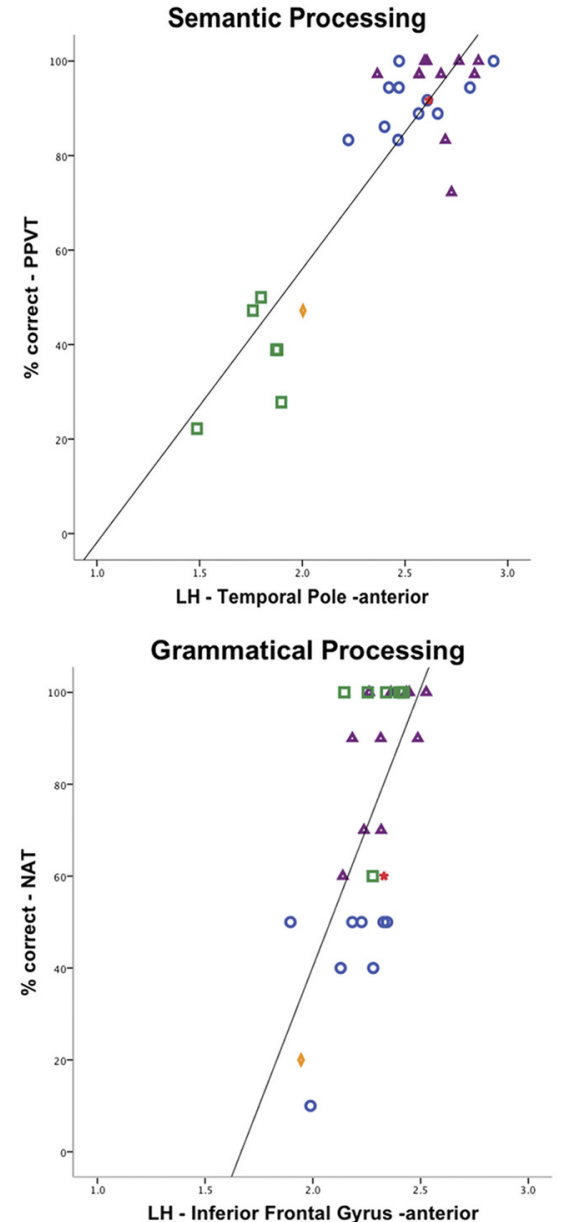

LH - Inferior Frontal Gyrus -anterior
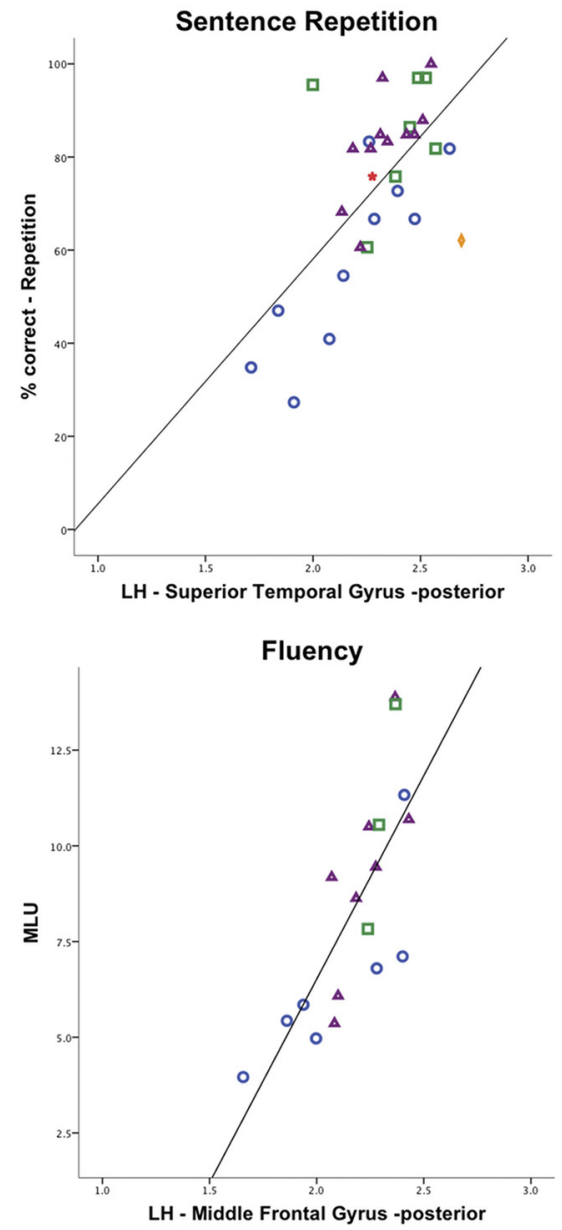

PPA-G $\square$ PPA-S A PPA-L PPA-M
Figure 3. Scatter plots illustrate the relationship between performance on a language measure and mean cortical thickness for the selected region. Different shapes are used to indicate the clinical subtype of each participant. Note, PPA-G/L refers to a patient with mixed features of the agrammatic and logopenic subtype. LH, Left hemisphere; PPA-M, mixed subtype; MLU, mean length of 
were most closely associated with the thinning of a discrete region in the medial part of the posterior superior temporal gyrus in the region of the supratemporal plane. The present results are consistent with those of Amici and colleagues (2007), who used voxel-based morphometry to demonstrate that gray matter loss in the left posterior portion of the superior temporal gyrus is associated with sentence repetition performance in a mixed group of clinical dementia patients (some with a diagnosis of PPA).

The strong correlation of the left anterior temporal lobe with semantic processing, as measured by single-word auditory comprehension scores in our study, was the most predictable finding, since numerous fMRI experiments in normal participants and meticulous clinical investigations of patients with the semantic variant of PPA have repeatedly shown the critical importance of this area for single-word comprehension (Hodges et al., 1992; Mummery et al., 1999; Rosen et al., 2002; Gorno-Tempini et al., 2004; Gitelman et al., 2005; Mesulam et al., 2009a,b). Our measure of semantic processing included items from several categories and did not examine the anatomical localization of categoryspecific deficits (e.g., living vs nonliving) shown by others (Brambati et al., 2006). One factor contributing to the strong correlation between single-word comprehension performance and the anterior temporal lobes may be the importance of visual pattern identification in the word-picture comprehension task we used. Although the stimuli in our single-word comprehension task were auditory word forms, the targets were visual, requiring cross-modal linkage of these representations. This feature of the task, the need to link a visually based object representation to an auditory word representation, may explain the strong correlation of performance in this task with atrophy in downstream visual and auditory association areas of the anterior temporal lobe (Mesulam, 2008).

Inspection of the correlation data by individual performance and subtype (Fig. 3) highlights that, although each subtype is characterized by the nature of the principal deficit, individual patients frequently display deficits in multiple language domains, each associated with cortical thinning (atrophy) in a characteristic set of regions. For example, Figure 3 demonstrates that reduced fluency rates were present in individual patients from each of the three subtypes and that performance on this task had a strong correlation $(p<0.001)$ with atrophy of the posterior middle frontal gyrus in the entire group of PPA patients, regardless of subtype. The scatter plots (Fig. 3) also demonstrate a continuum of repetition deficits within the whole group of patients, regardless of subtype associated with atrophy in the posterior superior temporal gyrus. Comprehension impairments of varying severity were seen in semantic as well as nonsemantic variants and were strongly correlated with atrophy in the anterior parts of the left hemisphere perisylvian language network and additional parts of the anterior temporal lobe. The fact that some PPA-G and PPA-L patients demonstrated mild word-comprehension performance deficits correlated with atrophy in the anterior temporal cortex suggests that atrophy in this area is not confined to the semantic subtype of PPA, but that it may also be seen in patients who best fit the criteria for other subtypes who have lesser word-comprehension deficits. Together, the structurefunction correlations identified in the present study demonstrate that subtle changes in cortical thickness measures have the sensitivity to capture subtle associations in decreased test performance, even in areas of impairment that are not the most salient in a given patient.
Some of the correlations in this study provide complementary information that cannot be obtained in patients with cerebrovascular lesions. For example, the type of damage caused by cerebrovascular lesions tends to obliterate dissociations between fluency and grammatical processing, whereas the partial and progressive loss of neurons in PPA allows for such dissociations to emerge in the course of the disease. The critical role of the anterior temporal lobes for naming and word comprehension has not been emphasized in investigations of stroke-related aphasia, probably because these areas are almost never damaged selectively by cerebrovascular accidents. Insights into this aspect of the language network emerged predominantly from investigations of patients with semantic subtypes of PPA (Hodges et al., 1992; Mummery et al., 1999; Rosen et al., 2002; Mesulam et al., 2009b). The study of PPA has, therefore, considerably enhanced our understanding of the language network.

\section{References}

Amici S, Ogar J, Brambati SM, Miller BL, Neuhaus J, Dronkers NL, GornoTempini ML (2007) Performance in specific language tasks correlates with regional volume changes in progressive aphasia. Cogn Behav Neurol 20:203-211.

Anderson JM, Gilmore R, Roper S, Crosson B, Bauer RM, Nadeau S, Beversdorf DQ, Cibula J, Rogish M 3rd, Kortencamp S, Hughes JD, Gonzalez Rothi LJ, Heilman KM (1999) Conduction aphasia and the arcuate fasciculus: a reexamination of the Wernicke-Geschwind model. Brain Lang 70:1-12.

Brambati SM, Myers D, Wilson A, Rankin KP, Allison SC, Rosen HJ, Miller BL, Gorno-Tempini ML (2006) The anatomy of category-specific object naming in neurodegenerative diseases. J Cogn Neurosci 18:1644-1653.

Damasio H, Damasio AR (1980) The anatomical basis of conduction aphasia. Brain 103:337-350.

Dickerson BC, Fenstermacher E, Salat DH, Wolk DA, Maguire RP, Desikan R, Pacheco J, Quinn BT, Van der Kouwe A, Greve DN, Blacker D, Albert MS, Killiany RJ, Fischl B (2008) Detection of cortical thickness correlates of cognitive performance: reliability across MRI scan sessions, scanners, and field strengths. Neuroimage 39:10-18.

Dunn L, Dunn D (2006) Peabody picture vocabulary test. Toronto, Onntario: Pearson Canada Assessment.

Fischl B, Dale AM (2000) Measuring the thickness of the human cerebral cortex from magnetic resonance images. Proc Natl Acad Sci U S A 97:11050-11055.

Fischl B, Sereno MI, Tootell RB, Dale AM (1999) High-resolution intersubject averaging and a coordinate system for the cortical surface. Hum Brain Mapp 8:272-284.

Fridriksson J, Kjartansson O, Morgan PS, Hjaltason H, Magnusdottir S, Bonilha L, Rorden C (2010) Impaired speech repetition and left parietal lobe damage. J Neurosci 30:11057-11061.

Genovese CR, Lazar NA, Nichols T (2002) Thresholding of statistical maps in functional neuroimaging using the false discovery rate. Neuroimage 15:870-878.

Gitelman DR, Nobre AC, Sonty S, Parrish TB, Mesulam MM (2005) Language network specializations: an analysis with parallel task designs and functional magnetic resonance imaging. Neuroimage 26:975-985.

Gorno-Tempini ML, Dronkers NF, Rankin KP, Ogar JM, Phengrasamy L, Rosen HJ, Johnson JK, Weiner MW, Miller BL (2004) Cognition and anatomy in three variants of primary progressive aphasia. Ann Neurol 55:335-346.

Hickok G, Erhard P, Kassubek J, Helms-Tillery AK, Naeve-Velguth S, Strupp JP, Strick PL, Ugurbil K (2000) A functional magnetic resonance imaging study of the role of left posterior superior temporal gyrus in speech production: implications for the explanation of conduction aphasia. Neurosci Lett 287:156-160.

Hillis AE (2007) Aphasia: progress in the last quarter of a century. Neurology 69:200-213.

Hodges JR, Patterson K, Oxbury S, Funnell E (1992) Semantic dementia: progressive fluent aphasia with temporal lobe atrophy. Brain 115:1783-1806.

Kertesz A (1982) Western aphasia battery. San Antonio: The Psychological Corporation. 
Lichtheim L (1885) On aphasia. Brain 7:433-484.

McCarthy RA, Warrington EK (1987) The double dissociation of shortterm memory for lists and sentences: evidence from aphasia. Brain 110:1545-1563.

Mesulam M (2008) Representation, inference, and transcendent encoding in neurocognitive networks of the human brain. Ann Neurol 64:367-378.

Mesulam MM (2003) Primary progressive aphasia: a language-based dementia. N Engl J Med 349:1535-1542.

Mesulam M, Wieneke C, Rogalski E, Cobia D, Thompson C, Weintraub S (2009a) Quantitative template for subtyping primary progressive aphasia. Arch Neurol 66:1545-1551.

Mesulam M, Rogalski E, Wieneke C, Cobia D, Rademaker A, Thompson C, Weintraub S (2009b) Neurology of anomia in the semantic variant of primary progressive aphasia. Brain 132:2553-2565.

Mummery CJ, Patterson K, Wise RJ, Vandenbergh R, Price CJ, Hodges JR (1999) Disrupted temporal lobe connections in semantic dementia. Brain 122:61-73.

Rohrer JD, Warren JD, Modat M, Ridgway GR, Douiri A, Rossor MN, Ourselin S, Fox NC (2009) Patterns of cortical thinning in the language variants of frontotemporal lobar degeneration. Neurology 72:1562-1569.

Rohrer JD, Ridgway GR, Crutch SJ, Hailstone J, Goll JC, Clarkson MJ, Mead S, Beck J, Mummery C, Ourselin S, Warrington EK, Rossor MN, Warren JD (2010) Progressive logopenic/phonological aphasia: erosion of the language network. Neuroimage 49:984-993.

Rosen HJ, Gorno-Tempini ML, Goldman WP, Perry RJ, Schuff N, Weiner M, Feiwell R, Kramer JH, Miller BL (2002) Patterns of brain atrophy in frontotemporal dementia and semantic dementia. Neurology 58:198-208.
Shaw P, Greenstein D, Lerch J, Clasen L, Lenroot R, Gogtay N, Evans A, Rapoport J, Giedd J (2006) Intellectual ability and cortical development in children and adolescents. Nature 440:676-679.

Sonty SP, Mesulam MM, Thompson CK, Johnson NA, Weintraub S, Parrish TB, Gitelman DR (2003) Primary progressive aphasia: PPA and the language network. Ann Neurol 53:35-49.

Thompson CK, Shapiro LP, Tait ME, Jacobs B, Schneider S, Ballard KJ (1995) A system for the linguistic analysis of agrammatic language production. Brain Lang 51:124-129.

Thompson CK, Ballard KJ, Tait ME, Weintraub S, Mesulam MM (1997) Patterns of language decline in non-fluent primary progressive aphasia. Aphasiology 11:297-321.

Walhovd KB, Fjell AM, Dale AM, Fischl B, Quinn BT, Makris N, Salat D, Reinvang I (2006) Regional cortical thickness matters in recall after months more than minutes. Neuroimage 31:1343-1351.

Weintraub S, Mesulam MM, Wieneke C, Rademaker A, Rogalski EJ, Thompson CK (2009) The northwestern anagram test: measuring sentence production in primary progressive aphasia. Am J Alzheimers Dis Other Demen 24:408-416.

Wright CI, Williams D, Feczko E, Barrett LF, Dickerson BC, Schwartz CE, Wedig MM (2006) Neuroanatomical correlates of extraversion and neuroticism. Cereb Cortex 16:1809-1819.

Wright CI, Feczko E, Dickerson B, Williams D (2007) Neuroanatomical correlates of personality in the elderly. Neuroimage 35:263-272.

Zatorre RJ, Evans AC, Meyer E, Gjedde A (1992) Lateralization of phonetic and pitch discrimination in speech processing. Science 256:846-849. 